\title{
Männlich, mittelalt, gebildet - oder? \\ Eine Charakterisierung deutschsprachiger Podcaster*innen
}

\author{
Christiane Attig, Technische Universität Chemnitz, Institut für Psychologie, \\ Angewandte Gerontopsychologie und Kognition, Wilhelm-Raabe-Str. 43, 09120 Chemnitz \\ E-Mail: christiane.attig@psychologie.tu-chemnitz.de
}

\begin{abstract}
Zusammenfassung
Podcasts erfahren nicht nur auf Seite der Hörenden eine immer stärkere Verbreitung. Auch die Zahl podcastproduzierender Personen steigt und macht Podcasts zu einem der aktuell wichtigsten partizipativen Medien. Zur Charakterisierung der Gruppe der Podcaster*innen liegen allerdings bisher nur wenige, überwiegend demographische Daten vor. Die vorliegende Arbeit soll daher dazu beitragen, die deutschsprachigen Podcastproduzierenden in drei Analysebereichen zu beschreiben: im Hinblick auf demographische, podcastspezifische und Persönlichkeitsvariablen. Mittels eines Online-Fragebogens wurden Daten von 653 Podcaster*innen gesammelt. Die Arbeit bietet erste Einblicke in die Diversität der deutschsprachigen Podcastproduzierenden und legt ein besonderes Augenmerk auf die Untersuchung von Geschlechterunterschieden.
\end{abstract}

Hinweis: Dies ist ein zur Veröffentlichung eingereichtes Manuskript. Änderungen durch den Peer Review-Prozess sind in dieser Version nicht berücksichtigt. Das Paper wird erscheinen in: kommunikation@gesellschaft, Sonderausgabe Podcasting (3. Quartal 2020), http://www.kommunikation-gesellschaft.de 


\section{Theoretischer Hintergrund}

Podcasts erfreuen sich seit den Anfängen vor etwa 15 Jahren stark steigender Popularität. Mit wachsender Zahl von Veröffentlichungen wächst auch die Zahl der Produzierenden, sowohl im Bereich privat und unabhängiger Podcastproduzierender als auch im Bereich privater und öffentlich-rechtlicher Medienunternehmen. Insbesondere die unabhängigen Podcaster*innen stellen aus medienpsychologischer Perspektive eine interessante Gruppe Medienschaffender dar, da diese dank niedrigschwelliger Einstiegshürden und dezentraler Veröffentlichungswege das partizipative Potenzial des Podcastmediums ausschöpfen (Lührmann 2019) und so in hohem Maße selbstbestimmt sog. user-generated content (McKenzie et al. 2012) produzieren. Ein Ergebnis dieser Autonomie ist ein hohes Maß an Themendiversität in Podcasts (Heise 2014). Die Genre- und Themenvielfalt wird zunehmend auch wissenschaftlich untersucht und reicht von Bildung (Drew 2017), über Gesellschaft und Kultur (Cwynar 2019), Wissenschaft (MacKenzie 2018), Perspektiven marginalisierter Gruppen (Tran 2019), True Crime (Boling 2019) bis hin zu (autobiographischen) Personal Podcasts, die die sprechende Person selbst in den Fokus rücken (Lindgren 2016).

Die Diversität der Podcastproduzierenden selbst wurde wissenschaftlich bisher kaum betrachtet. Obwohl angesichts der Varianz hinsichtlich bearbeitetem Thema und Professionalisierungsgrad der Produktion davon auszugehen ist, dass sich die Gruppe podcastproduzierender Personen durch ausgeprägte Vielfalt in demographischer und persönlicher Hinsicht auszeichnet, wurden in den bisherigen empirischen Studien nur wenige Daten zur Charakterisierung der Podcaster*innen präsentiert. Die Teilnehmenden in einer internationalen Studie mit über 1000 unabhängigen Podcastenden (Mocigemba/Riechmann 2007) waren im Median 34 Jahre alt, $13.8 \%$ davon weiblich und $54.8 \%$ verfügten über einen Hochschul- oder Universitätsabschluss. Ähnliche Daten lieferte Markman (2011) in einer Studie mit 135 Podcastenden (davon $12.2 \%$ weiblich, $40 \%$ im Alter zwischen 35 und 44, hohes Bildungsniveau). Im Rahmen einer Folgestudie mit 120 Podcastenden berichteten Markman und Sawyer (2014) vergleichbare demographische Daten, wobei der Anteil an Podcasterinnen höher war als zuvor (17.5 \% weiblich, im Durchschnitt 41 Jahre alt, hohes Bildungsniveau). Außerdem zeichneten sich die Podcastenden durch eine intensive Nutzung sozialer Medien aus (Markman 2011, Markman/Sawyer 2014). Persönlichkeitsvariablen wurden in keiner der bisherigen Arbeiten erhoben, sodass die Frage, wer eigentlich podcastet, nur unvollständig beantwortet werden kann.

Abgesehen von der Teilstichprobe in Mocigemba/Riechmann (2007) liegen außerdem für den deutschsprachigen Raum keine weiteren systematischen Befunde zur Charakterisierung podcastender Personen vor. Die vorliegende Arbeit nutzt einen explorativen Ansatz, um erste umfassende Einblicke in die Frage zu gewähren, durch welche demographischen, podcastspezifischen und Persönlichkeitsvariablen deutschsprachige Podcastende gekennzeichnet sind. Ein besonderes Augenmerk liegt auf der Analyse von 
Gerschlechterunterschieden, die bisher - auch aufgrund zu geringer Stichprobengrößen nicht untersucht, in der deutschsprachigen Podcastszene jedoch stark diskutiert wurden (z.B. Heise 2019).

Als Ausgangspunkt für die Analyse von Persönlichkeitsmerkmalen dienen die Big FivePersönlichkeitsdimensionen, auf denen die Persönlichkeit einer Person umfassend abgebildet werden kann (McCrae/John 1992). Als weitere relevante Persönlichkeitsmerkmale wurden Need for Cognition (NFC, Cacioppo/Petty 1982), interaktionsbezogene Technikaffinität (Affinity for Technology Interaction, ATI, Franke et al. 2019) sowie die Offenheit des Kommunikationsverhaltens (Mortensen et al. 1977) identifiziert. NFC oder "Denkfreude“ beschreibt die intrinsische Motivation, sich kognitiv anspruchsvollen Aufgaben zu widmen (Cacioppo/Petty 1982). In vielen Podcastproduktionen setzen sich die Podcastenden intensiv und zeitaufwendig mit verschiedensten Themen auseinander, sodass ein eher hoher NFCMittelwert in der Stichprobe erwartet wird. Podcasting bietet außerdem viele Ansatzpunkte für technikaffine Personen, sich mit Aufnahmegeräten und -software auseinanderzusetzen, sodass ein eher hoher ATI-Wert erwartet wird. Weiterhin ist Podcasten auch eine verbale Tätigkeit, von der sich wahrscheinlich Personen mit einem offenen Kommunikationsverhalten eher angesprochen fühlen, daher wird auch hier ein höherer Wert in der Stichprobe erwartet. Zuletzt wird die politische Einstellung der Podcastenden erfasst, um zu überprüfen, ob sich diese eher einem bestimmten politischen Spektrum zuordnen lassen.

\section{Methode}

\section{Stichprobe}

Durch die Online-Fragebogenstudie wurden von 654 Personen vollständige Datensätze gesammelt. Eine Person gab an, die Fragen nicht wahrheitsgemäß beantwortet zu haben; der dieser Person zugehörige Datensatz wurde von allen Analysen ausgeschlossen (also $N=653$ ). Es wurden Angaben zu insgesamt 940 Podcasts gemacht.

Die Rekrutierung der Teilnehmenden erfolgte von Mai-Oktober 2019 über E-Mail, über Aufrufe in Twitter sowie im Podcast-Forum Sendegate ${ }^{1}$ und über die Ansprache potenzieller Teilnehmender in einem Podcastprojekt der Autorin (Frede et al. 2019). Für die Ermittlung der E-Mail-Adressen wurde auf eine öffentlich zugängliche Liste der in Apple Podcasts verzeichneten deutschsprachigen Podcasts zurückgegriffen (Primbs 2019). Auf Basis dieser Liste wurden die Podcast-Feed-URLs automatisiert ausgelesen, die dort hinterlegten E-MailAdressen ermittelt und in einer Excel-Liste gespeichert. Diese Liste wurde nach Versendung der Rekrutierungs-Mails an insgesamt 8448 Empfänger*innen gelöscht. Die Teilnahme

\footnotetext{
${ }^{1}$ https://sendegate.de (Stand: 27.02.2020)
} 
dauerte etwa 45 Minuten. Die Teilnehmenden erhielten keine Vergütung für die Studienteilnahme.

\section{Material}

\section{Podcasting-Variablen}

Die Personen gaben zunächst die Anzahl der produzierten Podcasts an. Anschließend wurden weitere Variablen zu max. drei Podcasts erhoben, wobei die Personen Angaben zu mindestens einem Podcast machen mussten. Zu den erhobenen Variablen gehörten das Genre (Vorgabe von 16 Genres nach der Einteilung auf Fyyd ${ }^{2}$ sowie die Option „Sonstiges“; Single Choice), Zahl der Hörenden („Wie viele Hörer*innen hat eine Podcastfolge im Durchschnitt?“; Optionen „Ich weiß nicht" und „Keine Angabe“), bisheriger Zeitraum der Produktion (,Seit wann machen Sie diesen Podcast?“; Angabe von Monat/Jahr), Veröffentlichungsrhythmus („Wie regelmäßig veröffentlichen Sie neue Folgen dieses Podcasts?“; Antwortmöglichkeiten: unregelmäßig, täglich, einmal oder mehrmals wöchentlich, 14-tägig, monatlich, seltener), Veröffentlichungsmodalität („Wie veröffentlichen Sie diesen Podcast?“; Antwortmöglichkeiten: RSS-Feed, Spotify, iTunes, Audible, sonstige Plattformen; Multiple Choice), Monetarisierung („Verdienen Sie Geld mit diesem Podcast?“; Antwortmöglichkeiten: Ja: durch Werbung; Ja: durch freiwillige finanzielle Unterstützung („Spenden“); Ja: da es eine Auftragsarbeit/Teil meines Jobs ist; Nein) ${ }^{3}$, Vor- und Nachbereitungszeit in Stunden („Wie viel Vor- und Nachbereitungszeit stecken Sie im Durchschnitt in eine einzelne Episode dieses Podcasts?“), Intensität der Vor- und Nachbereitung („Wie würden Sie diesen Podcast auf einer Skala von ,reiner ,Laberpodcast', keine inhaltliche Vorbereitung' bis ,redaktionell aufwendig vor- und nachbereitet' einordnen?“; Beantwortung auf 7-stufiger Ratingskala von 1 minimale Vor- und Nachbereitung bis 7 - intensive Vor- und Nachbereitung) und Stärke der Hörerinteraktion („Wie würden Sie die Stärke der Hörerinteraktion für diesen Podcast einschätzen (z.B. hinsichtlich Feedback, Diskussionen, Hörertreffen)?“; Beantwortung auf 7stufiger Ratingskala von 1-gar keine Interaktion bis 7 - sehr intensive Interaktion).

Die wahrgenommene Zugehörigkeit zu einer Hörercommunity wurde mit zwei Items erfasst, die ebenfalls auf einer 6-stufigen Likert-Skala von 1 - stimmt gar nicht bis 6 - stimmt völlig beantwortet wurden (Beispielitem: „Ich fühle mich mit meinen Hörer*innen verbunden“). Die interne Konsistenz war akzeptabel $(\alpha=.76) .{ }^{4}$ Die wahrgenommene Zugehörigkeit zu einer Podcast-Community wurde mit sechs Items erfasst, die auf einer 6-stufigen Likert-Skala von 1 - stimmt gar nicht bis 6-stimmt völlig beantwortet wurden (Beispielitem: „Ich fühle mich als Teil einer Podcast-Community“). Die interne Konsistenz war akzeptabel ( $\alpha=.77$ ).

\footnotetext{
${ }^{2}$ https://fyyd.de (Stand 06.05.2019)

${ }^{3}$ Dieses Item wurde zunächst via Single Choice erfasst. Nachdem eine Person im freien Bemerkungsfeld anmerkte, dass ihr Podcast auf mehrere Arten monetarisiert wird, wurde dieses Item auf Multiple Choice umgestellt.

${ }^{4}$ Die Interpretation der internen Konsistenz erfolgt nach den gängigen Konventionen (siehe z.B. Cripps 2017).
} 


\section{Persönlichkeitsvariablen}

Zur Erfassung der Big Five-Persönlichkeitsvariablen wurde der BFI-10 (Rammstedt/John 2007) eingesetzt. Dieser erfasst die fünf Dimensionen mit jeweils zwei Items auf einer 5-stufigen Likert-Skala von 1 - trifft überhaupt nicht zu bis 5 - trifft voll und ganz zu. Die interne Konsistenz ist bei sehr kurzen Skalen, die breite Konstrukte erfassen sollen, kein geeignetes Maß zur Reliabilitätsbestimmung. Daher verweise ich auf Rammstedt/John (2007), die gezeigt haben, dass der BFI-10 über eine zufriedenstellende Retest-Reliabilität verfügt.

ATI wurde durch die ATI-Kurzskala (Wessel et al. 2019) auf einer 6-stufigen Likert-Skala von 1 - stimmt gar nicht bis 6 - stimmt völlig erfasst. Die interne Konsistenz war exzellent $(\alpha=.92)$. NFC wurde durch die NFC-Kurzskala (Beißert et al. 2015) auf einer 7-stufigen Likert-Skala von 1 - trifft überhaupt nicht zu bis 7 - trifft ganz genau zu beantwortet. Die interne Konsistenz war fragwürdig $(\alpha=.60)$.

Das Kommunikationsverhalten wurde durch die Verbal Predispositions Scale (Mortensen et al. 1977) erfasst. Die Items wurden von zwei Personen unabhängig voneinander ins Deutsche übersetzt. Abweichende Übersetzungen wurden diskutiert und harmonisiert. Der Fragebogen wurde auf einer 7-stufigen Likert-Skala von 1-trifft überhaupt nicht zu bis 7-trifft ganz genau $z u$ beantwortet. Die interne Konsistenz war exzellent $(\alpha=.93)$.

Die politische Einstellung wurde mit der Left-Right Self-Placement Scale (Breyer 2015) erfasst. Das Einzelitem wurde auf einer 11-stufigen Skala von 0 - rechts bis 10 - links beantwortet.

\section{Demographische Variablen}

Die folgenden demographischen Variablen wurden erfasst: Alter, soziales Geschlecht (weiblich, männlich, divers, keine Angabe) und aktuell höchster Bildungsabschluss (aktuell noch in Ausbildung, abgeschlossene Berufsausbildung, Meister/Fachschule, Fachhochschule/Universität, ohne Abschluss).

\section{Ergebnisse}

\section{Demographische Variablen}

Deskriptive Statistiken sind in Tabelle 1 zu finden. Die Podcaster*innen in der Stichprobe waren im Schnitt 38 Jahre alt, 137 Personen davon (21.0\%) $\leq 30$ Jahre, 261 (40.0\%) zwischen 31-40 Jahre, 188 (28.8 \%) zwischen 41- 50 Jahre und 67 (10.3\%) > 50 Jahre. In der Stichprobe identifizierten sich 164 Personen (25.1\%) als weiblich und 479 (73.4\%) als männlich (10 Personen $1.5 \%$ machten keine Angabe; niemand wählte die Option divers).

Die Stichprobe zeichnete sich außerdem durch ein hohes Bildungsniveau aus: Als höchsten Bildungsabschluss gaben 160 Personen (24.5\%) eine abgeschlossene Berufsausbildung an, 22 
(3.4 \%) Meister/Fachschulabschluss und 426 (65.2 \%) Fachhochschul-/Universitätsabschluss. Die restlichen Personen (45 bzw. 6.9 \%) verfügten (noch) über keinen Abschluss.

Tabelle 1: Deskriptive Statistiken der erhobenen Variablen.

\begin{tabular}{|c|c|c|c|c|c|c|c|c|}
\hline & $M$ & $S D$ & Min & Max & $\begin{array}{c}25 . \\
\text { Perzentil }\end{array}$ & $\begin{array}{c}50 . \\
\text { Perzentil } \\
\text { (Median) }\end{array}$ & $\begin{array}{l}75 . \\
\text { Perzentil }\end{array}$ & $n$ \\
\hline Alter & 38.22 & 8.91 & 19 & 67 & 31.50 & 38.00 & 44.00 & 653 \\
\hline $\begin{array}{l}\text { Anzahl produzierter } \\
\text { Podcasts }\end{array}$ & 1.65 & 2.05 & 1.00 & 40 & 1.00 & 1.00 & 2.00 & 653 \\
\hline $\begin{array}{l}\text { Geschätzte Anzahl der } \\
\text { Hörer*innen pro Episode }\end{array}$ & 2441.99 & 9400.98 & 0.00 & 140000.00 & 100.00 & 300.00 & 1000.00 & 815 \\
\hline Zeitraum der bisherigen & & & & & & & & \\
\hline $\begin{array}{l}\text { Podcastproduktion in } \\
\text { Monaten }\end{array}$ & 31.95 & 33.60 & 0.01 & 211.53 & 8.67 & 20.50 & 42.77 & 934 \\
\hline $\begin{array}{l}\text { Vor- und Nachbereitungszeit } \\
\text { in Stunden }\end{array}$ & 6.01 & 17.82 & 0.00 & 500.00 & 2.00 & 3.00 & 6.00 & 940 \\
\hline $\begin{array}{l}\text { Intensität der Vor- und } \\
\text { Nachbereitung }\end{array}$ & 4.15 & 1.60 & 1.00 & 7.00 & 3.00 & 4.00 & 5.00 & 940 \\
\hline Stärke der Hörerinteraktion & 2.91 & 1.43 & 1.00 & 7.00 & 2.00 & 3.00 & 4.00 & 940 \\
\hline Wahrgenommene & & & & & & & & \\
\hline $\begin{array}{l}\text { Zugehörigkeit zu einer } \\
\text { Hörer-Community }\end{array}$ & 4.00 & 1.19 & 1.00 & 6.00 & 3.00 & 4.00 & 5.00 & 653 \\
\hline Wahrgenommene & & & & & & & & \\
\hline $\begin{array}{l}\text { Zugehörigkeit zu einer } \\
\text { Podcast-Community }\end{array}$ & 3.25 & 1.09 & 1.00 & 6.00 & 2.50 & 3.17 & 4.00 & 653 \\
\hline Offenheit für Erfahrungen & 4.01 & 0.83 & 1.00 & 5.00 & 3.50 & 4.00 & 5.00 & 653 \\
\hline Gewissenhaftigkeit & 3.50 & 0.89 & 1.00 & 5.00 & 3.00 & 3.50 & 4.00 & 653 \\
\hline Extraversion & 3.40 & 1.04 & 1.00 & 5.00 & 2.50 & 3.50 & 4.00 & 653 \\
\hline Verträglichkeit & 3.24 & 0.78 & 1.00 & 5.00 & 3.00 & 3.00 & 4.00 & 653 \\
\hline Neurotizismus & 2.55 & 0.93 & 1.00 & 5.00 & 2.00 & 2.50 & 3.00 & 653 \\
\hline $\begin{array}{l}\text { Interaktionsbezogene } \\
\text { Technikaffinität }\end{array}$ & 4.11 & 1.36 & 1.00 & 6.00 & 3.00 & 4.50 & 5.25 & 653 \\
\hline Need for Cognition & 4.89 & 1.01 & 1.75 & 7.00 & 4.25 & 5.00 & 5.75 & 653 \\
\hline Kommunikationsverhalten & 4.38 & 0.86 & 1.68 & 6.52 & 3.82 & 4.44 & 5.00 & 653 \\
\hline Politische Einstellung & 2.52 & 1.57 & 0.00 & 10.00 & 1.50 & 2.00 & 3.00 & 653 \\
\hline
\end{tabular}




\section{Podcast-Variablen}

Bezüglich der Anzahl produzierter Podcasts gaben $68 \%$ an, einen Podcast zu produzieren (19 $\% 2$ Podcasts, 8 \% 3 Podcasts). Fünf Prozent der Teilnehmenden gaben an, > 3 Podcasts zu produzieren; das Maximum lag bei 40. Die Podcaster*innen wählten in Bezug auf 818 Podcasts eines der 16 vorgegebenen Genres; für die restlichen 122 Podcasts wurde die Option „Sonstiges“ plus Freitextantwort gewählt. Die Freitextantworten konnten in 83 der 122 Fälle jeweils einem der 16 vorgegebenen Genres zugeordnet werden. Die restlichen 39 Genreangaben (z.B. Persönlichkeitsentwicklung, Selbsthilfe) wurden in dem Genre „Weiterbildung/Ratgeber" zusammengefasst. Die finalen Prozentangaben zu den Podcastgenres lassen sich in Abbildung 1 finden. Die am häufigsten genannten Genres sind somit Gesellschaft/Kultur, Spiele/Hobbies sowie Wissen/Wissenschaft.

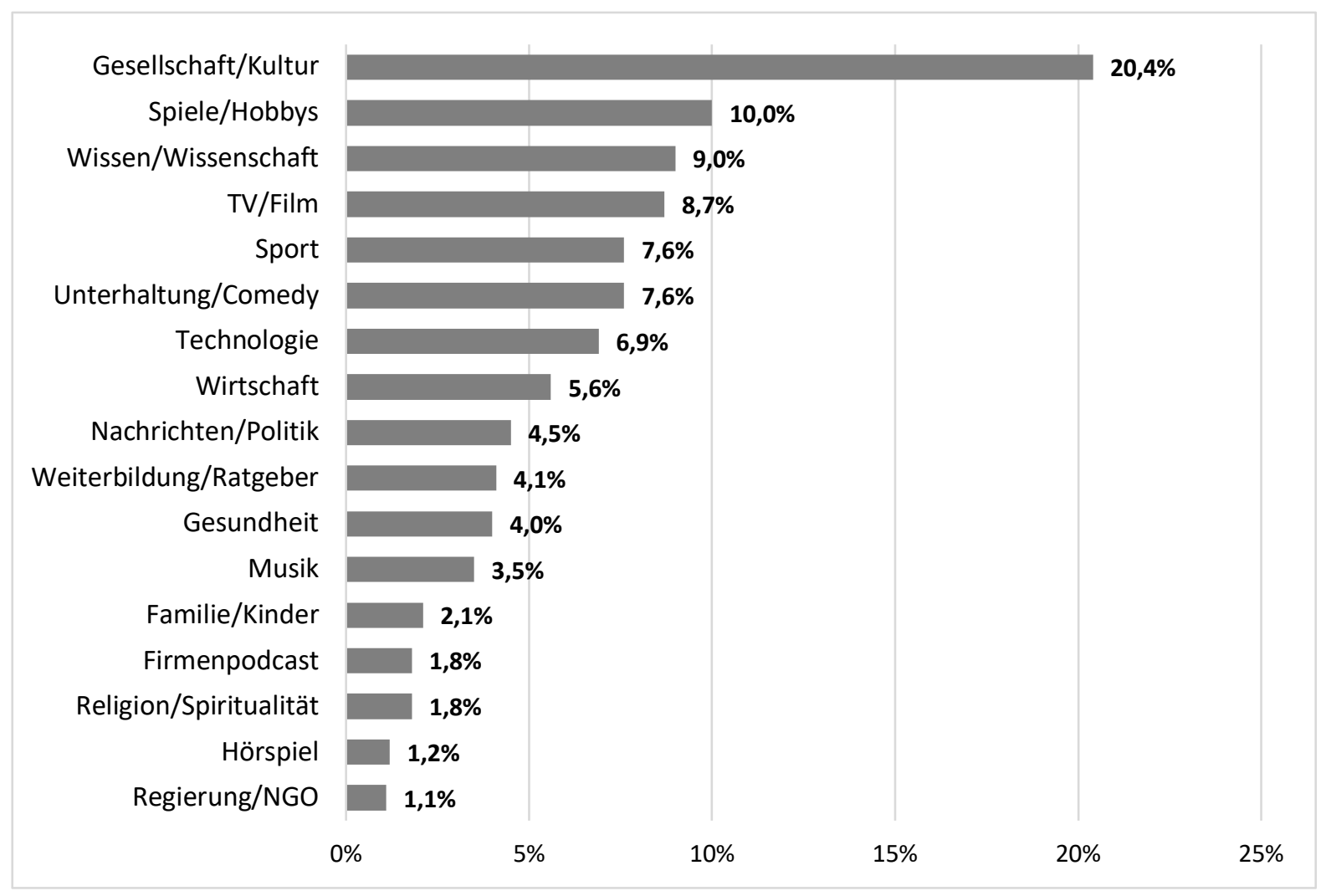

\section{Abbildung 1: Bearbeitete Podcastgenres in der Stichprobe.}

Eine Angabe zur geschätzten Zahl der Hörer*innen wurde in Bezug auf 815 Podcasts gemacht. Der Mittelwert lag bei 2442 Hörer*innen (Spannweite $=140000$ ), wobei die Mehrheit deutlich kleinere Zahlen angab (für 613 Podcasts/75.3 \% wurden $\leq 1000$ Hörer*innen/Folge und für lediglich 54 Podcasts/6.6 \% $\geq 10000$ Hörer*innen/Folge angegeben).

Zur Frage, seit wann sie den Podcast produzieren, gaben sechs Personen ein Datum in der Zukunft an; diese Fälle wurden aus der Analyse dieser Variable ausgeschlossen (daher $n=934$ ). Der Mittelwert des bisherigen Produktionszeitraums lag bei 32 Monaten und zeigte eine große Varianz (Spannweite $=212$ Monate). Dreihundertzehn Podcasts (33.2 \%) wurden seit $<1 \mathrm{Jahr}$ 
produziert, 193 (20.7 \%) Podcasts seit 1-2 Jahren, 142 (15.2 \%) Podcasts seit 2-3 Jahren, 144 Podcasts (15.4\%) seit 3-5 Jahren und 145 (15.5\%) > 5 Jahre.

Achthundertfünfunddreißig der 940 Podcasts (88.8\%) wurden über Apple Podcasts publiziert, $824(87.7 \%)$ via RSS-Feed, 633 (67.3\%) über Spotify und 641 (68.2 \%) über sonstige Plattformen (z.B. Deezer, Soundcloud, Youtube, Audible). Acht Podcasts (0.9\%) wurden täglich veröffentlicht, 226 (24.0 \%) ein- oder mehrmals in der Woche, 215 (22.9 \%) 14-tägig, 180 (19.1\%) monatlich, 55 (5.9\%) seltener als monatlich und 256 (27.2 \%) unregelmäßig.

Siebenhunderteinundvierzig Podcasts $(76.8$ \%) wurden nicht monetarisiert. Einhundertvierzehn (11.8\%) der Podcasts wurden durch freiwillige finanzielle Unterstützung monetarisiert und 39 (4.0\%) durch Werbeeinnahmen. Die Stichprobe bestand außerdem zum größten Teil aus unabhängigen, privat produzierenden Podcastenden, denn nur 71 Podcasts (7.4 \%) waren Auftragsproduktionen oder Teil der Erwerbstätigkeit.

Hinsichtlich der Vor- und Nachbereitungszeit für eine einzelne Podcastepisode lag der Durchschnitt bei 6 Stunden, wobei die Varianz enorm war (Spannweite $=500 \mathrm{Std}$ ). Die Ergebnisse zeigen, dass 334 Podcasts $(35.5 \%) \leq 2$ Stunden Vor- und Nachbereitungszeit pro Folge benötigten, 250 (26.6 \%) 2-4 Stunden, 254 (27.0\%) 4-10 Stunden und 102 (10.9\%) > 10 Stunden.

Die Stärke der Interaktion mit den Hörer*innen wurde als eher gering eingeschätzt; der Mittelwert wich signifikant vom Skalenmittelwert 4 nach unten ab $(M=2.91, t(939)=-23.33$, $p<.001, d=-0.76$, mittlerer bis großer Effekt ${ }^{5}$ ). Dennoch gaben die Podcastenden im Schnitt an, dass sie mit ihren Hörer*innen verbunden fühlen; der Mittelwert wich signifikant vom Skalenmittelwert 3.5 nach oben $\mathrm{ab}(M=4.00, t(652)=10.86, p<.001, d=0.42$, kleiner Effekt). Beide Werte korrelieren positiv, d.h., je stärker die wahrgenommene Interaktion mit den Hörenden, desto höher das Verbundenheitsgefühl ( $r=.51, p<.001$, mittlerer Effekt). Die wahrgenommene Zugehörigkeit zu einer Podcast-Community wurde signifikant niedriger als die zu den Hörenden eingeschätzt $(M=3.25, t(652)=14.21, p<.001, d=0.66$, mittlerer Effekt).

\section{Persönlichkeitsvariablen}

Hinsichtlich der Big Five zeigte sich ein ausgeprägter Wert auf der Offenheits-Dimension, der deutlich von Skalenmittelwert 3 abwich $(M=4.01, t(652)=33.69, p<.001, d=1.32$, großer Effekt). Kleine bis mittelgroße Abweichungen nach oben zeigten sich außerdem in Bezug auf Gewissenhaftigkeit $(M=3.50, t(652)=14.48, p<.001, d=0.57)$, Extraversion $(M=3.40, t(652)$ $=9.80, p<.001, d=0.38)$ und Verträglichkeit $(M=3.24, t(652)=7.74, p<.001, d=0.30)$. Der Mittelwert für Neurotizismus wich dagegen signifikant vom Skalenmittelwert nach unten ab $(M=2.55, t(652)=-12.45, p<.001, d=-0.49$, kleiner bis mittlerer Effekt).

Die Podcaster*innen in der Stichprobe waren eher hoch technikaffin; der Mittelwert wich signifikant vom Skalenmittelwert 3.5 nach oben ab $(M=4.11, t(652)=11.43, p<.001, d=0.44$,

\footnotetext{
${ }^{5}$ Die Interpretation der Effektstärken erfolgt nach den Konventionen von Cohen (1992).
} 
kleiner Effekt). Ähnlich verhielt es sich mit NFC, wobei der Effekt stärker ausgeprägt war ( $M=$ 4.89, $t(652)=22.43, p<.001, d=0.88$, großer Effekt). Des Weiteren zeichnete sich die Stichprobe durch ein eher offenes Kommunikationsverhalten aus; der Mittelwert wich signifikant vom Skalenmittelwert 4 nach oben ab $(M=4.38, t(652)=11.31, p<.001, d=0.44$, kleiner Effekt).

Bezüglich der politischen Einstellung ordnete sich die große Mehrheit der Podcaster*innen in der Stichprobe dem linken Spektrum zu; 576 Personen (88.2 \%) kreuzten auf der 11-stufigen Skala Werte zwischen 0 und 4 an. Die Abweichung vom Skalenmittelwert 5 war signifikant und deutlich ausgeprägt $(M=2.52, t(652)=-40.38, p<.001, d=-1.58$, großer Effekt).

\section{Geschlechterunterschiede}

Aufgrund der unterschiedlichen Gruppengrößen in Verbindung mit teilweisen Verletzungen der Voraussetzung der Homoskedastizität wurden sowohl parametrische als auch nonparametrische Tests zur Analyse von Geschlechterunterschieden durchgeführt. Da diese nicht zu unterschiedlichen Ergebnissen führten, werden folgend nur die Ergebnisse der parametrischen Tests berichtet.

Die Podcasterinnen produzierten im Schnitt weniger Podcasts als Podcaster $\left(M_{\mathrm{w}}=1.24\right.$ vs. $M_{\mathrm{m}}$ $=1.71, t(632.41)=-5.37, p<.001, d=0.34$, kleiner Effekt). Außerdem produzierten sie diese seit einem kürzeren Zeitraum $\left(M_{\mathrm{w}}=21.80\right.$ Monate vs. $M_{\mathrm{m}}=36.65$ Monate, $t(488.09)=-6.38$, $p<.001, d=0.45$, kleiner Effekt). Keine signifikanten Geschlechterunterschiede ergaben sich hinsichtlich Alter, Anzahl der Hörer*innen, Vor- und Nachbereitungszeit, Intensität der Vorund Nachbereitung, Intensität der Hörerinteraktion und Zugehörigkeitsgefühl zu Hörer*innen- und Podcastcommunity.

Bezüglich der Podcastgenres zeigten sich auf deskriptiver Ebene einige Unterschiede (siehe Abbildung 2). Von den Podcasterinnen in der Stichprobe gaben $27.6 \%$ als primäres bearbeitetes Podcastgenre Gesellschaft/Kultur an; von den Podcastern waren es $17.1 \%$. Weitere Genres, die relativ betrachtet eher von Frauen bearbeitet werden ${ }^{6}$, waren Gesundheit (11.7 vs. $2.7 \%$ ), Weiterbildung/Ratgeber (11.0 vs. $2.9 \%$ ), Familie/Kinder (3.7 vs. $1.5 \%)$ und Wirtschaft (8.0 vs. $6.1 \%$ ). Genres, die eher von Podcastern bearbeitet werden, waren Technologie (8.6 vs. $1.2 \%$ ), Sport (9.2 vs. $2.5 \%$ ), TV/Film (7.9 vs. $2.5 \%$ ), Spiele/Hobbys (12.3 vs. $7.4 \%$ ), Nachrichten/Politik (5.2 vs. $1.8 \%$ ) und Hörspiel (1.7 vs. $0.0 \%$ ). Keine bzw. kaum (< $1.5 \%) \quad$ Unterschiede zeigten sich hinsichtlich Wissen/Wissenschaft, Unterhaltung/Comedy, Musik, Religion/Spiritualität, Firmenpodcast und Regierung/NGO.

\footnotetext{
${ }^{6}$ Aufgrund der ungleichen Gruppengrößen wurden in der Stichprobe alle Podcastgenres (außer Gesundheit) in absoluten Zahlen von mehr Podcastern als Podcasterinnen angegeben.
} 


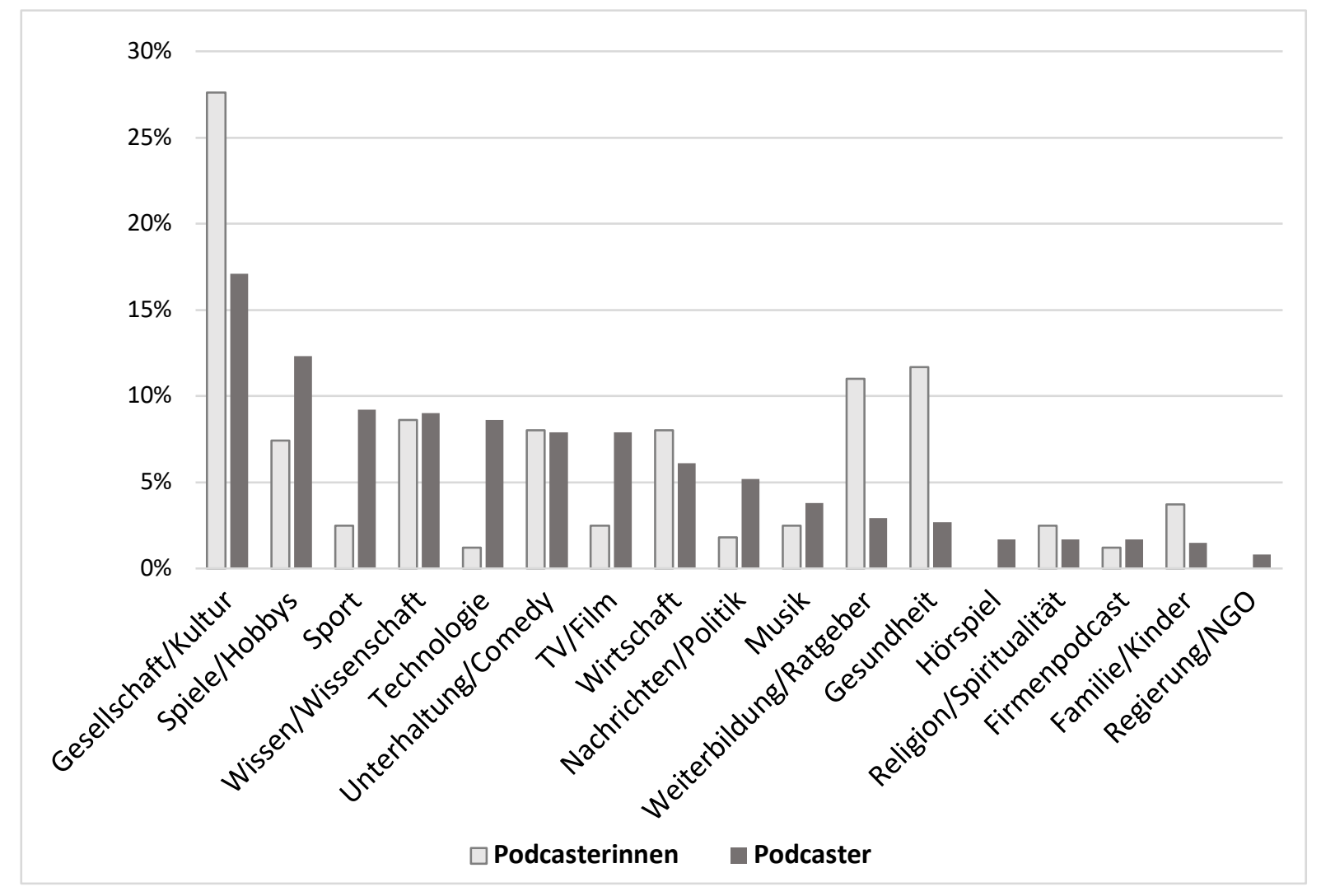

Abbildung 2: Bearbeitete Podcastgenres nach Geschlecht.

Der Anteil der Podcaster*innen, die ihren Podcast monetarisieren, unterschied sich kaum zwischen den Geschlechtern (w: 18.9\%, m: $21.1 \%$ ). Werbeeinnahmen erzielen ebenfalls etwa gleich viele Podcaster*innen (w: $4.9 \%, \mathrm{~m}$ : 4.6\%). Es werden jedoch mehr Podcaster freiwillig finanziell unterstützt (w: $8.5 \%$, m: 14.8\%). Ein größerer relativer Anteil an Podcasterinnen gab im Gegensatz dazu an, dass die Podcastproduktion eine Auftragsarbeit/Teil der Erwerbstätigkeit sei (w: $7.9 \%$, m: $4.8 \%$ ).

Die Podcasterinnen in der Stichprobe hatten hinsichtlich aller fünf Big FivePersönlichkeitsdimensionen signifikant höhere Mittelwerte als die Podcaster (kleine bis mittlere Effekte): Gewissenhaftigkeit $\left(M_{\mathrm{w}}=3.82\right.$ vs. $M_{\mathrm{m}}=3.39, t(329.25)=5.95, p<.001, d=$ $0.54)$, Extraversion $\left(M_{\mathrm{w}}=3.76\right.$ vs. $\left.M_{\mathrm{m}}=3.28, t(303.55)=5.41, p<.001, d=0.47\right)$, Offenheit $\left(M_{\mathrm{w}}=4.32\right.$ vs. $\left.M_{\mathrm{m}}=4.02, t(338.35)=4.40, p<.001, d=0.36\right)$, Verträglichkeit $\left(M_{\mathrm{w}}=3.43\right.$ vs. $\left.M_{\mathrm{m}}=3.17, t(287.09)=3.87, p<.001, d=0.35\right)$, Neurotizismus $\left(M_{\mathrm{w}}=2.73 \mathrm{vs.} M_{\mathrm{m}}=2.48, t(641)\right.$ $=3.83, p=.004, d=0.27)$.

Darüber hinaus zeichneten sich die Podcasterinnen durch eine signifikant geringere ATI $\left(M_{\mathrm{w}}=\right.$ 3.26 vs. $M_{m}=4.39, t(262.00)=-9.42, p<.001, d=0.89$, großer Effekt), ein offeneres Kommunikationsverhalten $\left(M_{\mathrm{w}}=4.60\right.$ vs. $M_{\mathrm{m}}=4.31, t(641)=3.71, p<.001, d=0.34$, kleiner Effekt) und eine noch progressivere (,linkere“) politische Einstellung aus als die Podcaster in der Stichprobe $\left(M_{\mathrm{w}}=2.24\right.$ vs. $M_{\mathrm{m}}=2.64, t(340.54)=-3.14, p=.002, d=0.26$, kleiner Effekt). In Bezug auf NFC gab es keine signifikanten Unterschiede. 


\section{Diskussion}

Die Ergebnisse zeigen im Vergleich zu bisherigen Befunden (Markman 2011; Markman/Sawyer 2014; Mocigemba/Riechmann 2007) einen größeren Anteil weiblicher Podcastender. Ein mittleres Durchschnittsalter und eher hohes Bildungsniveau wurden auch hier gefunden. Darüber hinaus konnte durch zahlreiche Podcast- und Persönlichkeitsvariablen ein präziseres Bild der deutschsprachigen Podcaster*innen gezeichnet werden: Der Großteil der Stichprobe produzierte einen Podcast (sog. Monocaster; Mocigemba/Riechmann 2007), monetarisiert den Podcast nicht und erreicht bis zu 1000 Hörer*innen pro Episode. Darüber hinaus zeichneten sie sich durch eine deutlich ausgeprägte Offenheit für Erfahrungen und Denkfreude aus und waren deutlich dem linken politischen Spektrum zuzuordnen. Dieser Befund untermauert bestehende Ergebnisse, denn der negative Zusammenhang zwischen Offenheit und Konservatismus wurde häufig nachgewiesen (z.B. Jost et al. 2003), ebenso wie der positive Zusammenhang zwischen Offenheit und NFC (z.B. Fleischhauer et al. 2009).

Die Analyse von Geschlechterunterschieden hat gezeigt, dass die Podcasterinnen in der Stichprobe seit kürzerer Zeit weniger Podcasts produzieren als Podcaster. Gemeinsam mit dem höheren Frauenanteil im Vergleich zu den vergangenen Studien deutet dies daraufhin, dass Podcasterinnen aufgeholt und die ursprünglich sehr maskulin geprägte Podcastszene stärker durchmischt haben. Der Unterschied in den Podcastgenres eröffnet die Frage, ob die stärker von Frauen besetzten Themen (z.B. Gesellschaft/Kultur, Weiterbildung/Ratgeber) in Zukunft insgesamt noch stärkere Berücksichtigung erfahren, falls der vermutete Aufwärtstrend der Podcasterinnen anhält. Die in allen Big Five-Dimensionen stärkeren Ausprägungen der Podcasterinnen sind, bis auf Extraversion, nicht ungewöhnlich (Rammstedt 2007). Ungewöhnlich ist auch nicht der geringere ATI-Wert der Podcasterinnen; dieser findet sich auch in der allgemeinen Bevölkerung (Franke et al. 2019). Deutlich wird allerdings, dass der insgesamt höhere ATI-Wert in der Stichprobe auf den deutlich höheren Wert der männlichen Podcastenden zurückgeht, welcher sich wiederum ebenfalls nicht deutlich vom dem der männlichen Personen in der allgemeinen Bevölkerung unterscheidet (Franke et al. 2019).

Als einschränkend muss die Stichprobenselektivität genannt werden. Aufgrund der persönlichen Ansprache der Autorin in sozialen Medien und im Podcast kann nicht ausgeschlossen werden, dass der Anteil an Podcasterinnen durch eine erhöhte Zahl persönlich bekannter weiblicher Podcastender verzerrt wurde oder sich diese durch mediale Berichterstattung über Podcasterinnen (z.B. Heise 2019) besonders zur Teilnahme motiviert wurden. Außerdem ist davon auszugehen, dass Podcastende, die gut in sozialen Medien miteinander vernetzt sind, schneller und häufiger auf die Studie aufmerksam wurden und in der Stichprobe überrepräsentiert sind.

Abschließend bietet die vorliegende Arbeit einen wertvollen Einblick in die Charakterisierung der (insbesondere unabhängigen) deutschsprachigen Podcaster*innen und bildet einen 
Grundstein für zukünftige Arbeiten, die sich noch tiefergehender mit medienpsychologischen Fragestellungen in Bezug auf das Podcasting beschäftigen werden.

\section{Literatur}

Beißert, Hanna, Meike Köhler, Marina Rempel und Constanze Beierlein, 2015, Kurzskala Need for Cognition NFC-K, Zusammenstellung sozialwissenschaftlicher Items und Skalen.

Boling, Kelli S., 2019, True crime podcasting: Journalism, justice or entertainment?, Radio Journal: International Studies in Broadcast \& Audio Media 17: 161-178.

Breyer, Bianka, 2015, Left-Right Self-Placement (ALLBUS), Zusammenstellung sozialwissenschaftlicher Items und Skalen.

Cacioppo, John T. und Richard E. Petty, 1982, The need for cognition, Journal of Personality and Social Psychology 42: 116-131.

Cohen, Jacob, 1992, A power primer, Psychological Bulletin 112: 155-159.

Cripps, Barry (Hg.), 2017, Psychometric testing: Critical perspectives. Chichester. John Wiley \& Sons.

Cwynar, Christopher, 2019, Self-service media: Public radio personalities, reality podcasting, and entrepreneurial culture, Popular Communication 17: 317-332.

Drew, Christopher, 2017, Educational podcasts: A genre analysis, E-Learning and Digital Media 14: 201-211.

Fleischhauer, Monika, Sören Enge, Burkhard Brocke, Johannes Ullrich, Alexander Strobel und Anja Strobel, 2009, Same or different? Clarifying the relationship of need for cognition to personality and intelligence, Personality and Social Psychology Bulletin 36: 82-96.

Franke, Thomas, Christiane Attig und Daniel Wessel, 2019, A personal resource for technology interaction: Development and validation of the Affinity for Technology Interaction (ATI) scale, International Journal of Human-Computer Interaction 35: 456-467.

Frede, Danny, Joscha Grunewald und Christiane Attig, 2019, Schwammintelligenz. 03.06.2019. Podcastepisode: https://hoerer.podigee.io/5-schwammintelligenz (Stand 27.02.2020)

Heise, Nele, 2014, On the shoulders of giants? How audio podcasters adopt, transform and re-invent radio storytelling, Transnational Radio Stories. MOOC des Masterstudiengangs Onlineradio an der MLU Halle-Wittenberg.

Heise, Nele, 2019, Das Beste, was Podcasts passieren konnte. Online-Publikation: https://neleheise.de/2019/11/19/das-beste-was-podcasts-passieren-konnte/

Lindgren, Mia, 2016, Personal narrative journalism and podcasting. The Radio Journal - International Studies in Broadcast \& Audio Media 14: 23-41.

Lührmann, Katharina, 2019, Podcasts als Raum politisch-medialer Kommunikation. Mannheim. Tectum. 
Jost, John T., Jack Glaser, Arie W. Kruglanski, und Jack J. Sulloway, 2003, Political conservatism as motivated social cognition, Psychological Bulletin 129: 339-375.

MacKenzie, Lewis E., 2019, Science podcasts: Analysis of global production and output from 2004 to 2018, Royal Society Open Science 6: 180932.

Markman, Kris M., 2011, Doing radio, making friends, and having fun: Exploring the motivations of independent audio podcasters, new media \& society 14: 547-565.

Markman, Kris M. und Caroline Elizabeth Sawyer, 2014, Why pod? Further explorations of the motivations for independent podcasting, Journal of Radio \& Audio Media 21: 20-35.

McKenzie, Pamela J., Jacquelyn Burkell, Lola Wong, Caroline Whippey, Samuel E. Trosow und Michael McNally, 2012, User-generated online content 1: Overview, current state and context. First Monday 17: 412-422.

Mocigemba, Dennis und Gerald Riechmann, 2007, International Podcastersurvey. Präsentation.

Mortensen, C. David., Paul H. Arntson und Myron Lustig, 1977, The measurement of verbal predispositions: Scale development and application, Human Communication Research 3: 146-158.

Primbs, Dirk, 2019, germanpodcasts. Google Spreadsheet:

https://docs.google.com/spreadsheets/d/10XMgzwP7t3NpcbaYRjNPgCIPwpsB8JhML77EmXfpUKY/e dit\#gid=407345763 (Stand 27.02.2020)

Rammstedt, Beatrice, 2007, The 10-Item Big Five Inventory, European Journal of Psychological Assessment 23: 193-201.

Rammstedt, Beatrice und Oliver P. John, 2007, Measuring personality in one minute or less: A 10item short version of the Big Five Inventory in English and German, Journal of Research in Personality 41: 203-212.

Tran, Tony, 2019, Creating sound in silences: The Second Wave podcast and pluralizing Vietnamese diasporic histories, Popular Communication, 17:4, 288-300.

Wessel, Daniel, Christiane Attig und Thomas Franke, 2019, ATI-S - An ultra-short scale for assessing affinity for technology interaction in user studies, S. 147-154, in: MuC'19: Proceedings of Mensch und Computer 2019.

\section{Danksagungen}

Ich danke Nicolas Wöhrl, Sandro Schroeder, den Mitgliedern des Podcast-Meetup Leipzig, insbesondere Jochen Dreier und Friedemann Brenneis sowie den Mitgliedern des Sendegates für ihr hilfreiches Feedback bei der Konzeption der Studie. Außerdem danke ich Kristina Buhl für ihre Unterstützung bei der Fragebogenentwicklung und Studiendurchführung, Martin Krumsdorf für seine Hilfe bei der Rekrutierung der Teilnehmenden und Oliver Vettermann für das Lektorat. Schließlich danke ich von Herzen allen Podcaster*innen, die an dieser Studie teilgenommen haben. 